\title{
Rapamycin modulated brain-derived neurotrophic factor and B-cell lymphoma 2 to mitigate autism spectrum disorder in rats
}

This article was published in the following Dove Press journal:

Neuropsychiatric Disease and Treatment

20 March 2017

Number of times this article has been viewed

\author{
Jie Zhang' \\ Li-Ming Liu' \\ Jin-Feng $\mathrm{Ni}^{2}$ \\ 'Department of Child Healthcare, \\ ${ }^{2}$ Department of Pediatrics, Maternal \\ and Children Hospital of Tangshan \\ City, Tangshan, Hebei, People's \\ Republic of China
}

\begin{abstract}
The number of children suffered from autism spectrum disorder (ASD) is increasing dramatically. However, the etiology of ASD is not well known. This study employed mammalian target of rapamycin inhibitor rapamycin to explore its effect on ASD and provided new therapeutic strategies for ASD. ASD rat model was constructed and valproic acid (VPA) was injected intraperitoneally into rats on pregnancy day 12.5. Offspring from VPA group were divided into ASD group and ASD + rapamycin (ASD + RAPA) group. Compared with normal group, the frequency and duration of social behavior and straight times of ASD group were shortened, but the grooming times were extended. Meanwhile, in ASD group, the average escape latency and the frequency of crossing plates were decreased, the apoptotic index (AI) detected by TUNEL assay was increased, and the expression of brain-derived neurotrophic factor (BDNF) and B-cell lymphoma 2 (Bcl-2) analyzed was decreased with great difference compared with normal group $(P<0.01)$. However, rapamycin treatment in ASD rats mitigated the ASD-like social behavior, such as the frequencies of straight and grooming. Furthermore, rapamycin shortened the average escape latency, but increased the frequency of crossing plates of ASD rats. In hippocampus, rapamycin decreased the AI, but increased the levels of BDNF and Bcl-2 $(P<0.01)$ of ASD rats. These findings revealed that rapamycin significantly mitigated the social behavior by enhancing the expression of BDNF and Bcl-2 to suppress the hippocampus apoptosis in VPA-induced ASD rats.
\end{abstract}

Keywords: autism spectrum disorder, B-cell lymphoma 2, brain-derived neurotrophic factor, hippocampus, rapamycin, valproic acid

\section{Introduction}

The term autism spectrum disorder (ASD) refers to a group of conditions characterized by pervasive impairments in social interaction, deficits in language and communication, and repetitive and stereotyped patterns of behaviors and interests. ${ }^{1}$ ASD usually develops in the first 3 years of life and has a serious impact on children's cognition, language, emotion, and social behavior. ${ }^{2}$ The number of children suffered from ASD is increasing dramatically. As we know, the pathogenesis of ASD is complex and may be associated with genetic factor, immune, environment, education, diet, and so on. ${ }^{3}$ However, the etiology of ASD is unclear, and an objective diagnostic criteria and treatment options are still lacking.

The phosphatidylinositol-3-kinase/phosphatase and tensin homolog (PTEN)/ mammalian target of rapamycin (mTOR) pathway regulates various neuronal functions. Tuberous sclerosis complex $1 / 2(\mathrm{TSC} 1 / 2)$ is a key factor in mTOR signaling pathway, and its protein products are essential in the regulation of mTOR activity. ${ }^{4}$
Department of Pediatrics, Maternal and Children Hospital of Tangshan City, 063000, No 14 South Road, Tangshan, Hebei, People's Republic of China

Email jinfengni2016@I63.com 
The relevance of mTOR signal is increasingly appreciated in human diseases, such as epilepsies and ASD. ${ }^{5,6}$ For example, the heterozygous mutations in the TSC1 or TSC2 substantially elevate an individual's risk to develop ASD. ${ }^{7}$ Thus, to investigate the role of mTOR signal in ASD is expected to help explore the molecular pathophysiology of ASD and potential pharmacological therapies. Rapamycin functions as an mTOR inhibitor by blocking the binding between mTOR and other protein components and by declining the phosphorylation of mTOR. ${ }^{8}$ Some evidence showed that rapamycin treatment protected neuron viability in stroke, parkinsonism, and early stage Alzheimer-type Tauopathy animal models. ${ }^{9-11}$ Strikingly, rapamycin supplement mitigated social behavioral abnormality in ASD animal model BTBR mice; ${ }^{12}$ however, the mechanism was not well revealed.

ASD is a neurodevelopmental disorder with the defect of learning and memory abilities. The hippocampus is revealed to modulate the learning and memory and spatial abilities. Several evidence highlights that hippocampal neurons are usually impaired in ASD animal. ${ }^{13}$ Exposure to valproic acid (VPA) during pregnancy has been linked with increased incidence of ASD. ${ }^{14}$ This study constructed the VPA-induced ASD rat model and used hippocampus as the target tissue to evaluate the effect of rapamycin supplement on learning and memory and to reveal the molecular mechanism by which rapamycin improved the impairment of hippocampus and stereotyped behavior in VPA-induced ASD model.

\section{Materials and methods}

\section{Animal culture}

In total, 48 adult healthy Wistar rats of half gender were purchased from Shanghai Silaike Laboratory Animal Limited Liability Company (Shanghai, China) (qualification certificates: SCXK [Hu] 2012-0002). Female rats weighed 200-250 g and male 300-350 g. The animals were kept at standard laboratory conditions on arrival at $23^{\circ} \mathrm{C} \pm 2^{\circ} \mathrm{C}$. Standard rodent feed and water were available ad libitum. All procedures were performed in accordance with the Guide for the Humane Use and Care of Laboratory Animals issued by Maternal and Children Hospital of Tangshan city.

\section{Drugs and reagents}

Rapamycin (HY-10219) and VPA were obtained from Sigma-Aldrich Co. (St Louis, MO, USA). VPA was dissolved in $0.9 \%$ saline at a concentration of $250 \mathrm{mg} / \mathrm{mL}$. Rabbit monoclonal anti-brain-derived neurotrophic factor (anti-BDNF), B-cell lymphoma 2 (Bcl-2), and mouse monoclonal anti-GAPDH antibodies were purchased from Santa Cruz Biotechnology Inc. (Dallas, TX, USA).

\section{Creation of ASD rat model by VPA exposure and animal grouping}

Sexually mature female and male rats were mated overnight after the estrous cycle was monitored. The female vaginal suppository was checked on the next morning, and rats with pessary were considered to be at pregnancy day 1 (E1). The pregnant rats were housed individually and randomly divided into two groups (control group and VPA group). Female rats in VPA group received the intraperitoneal injection of VPA $(600 \mathrm{mg} / \mathrm{kg}, 250 \mathrm{mg} / \mathrm{mL}$ diluted in $0.9 \% \mathrm{NaCl})$ on $\mathrm{E} 12.5$, and female rats in control group were injected with physiological saline at the same time according to the previous description. ${ }^{14}$ The offspring rats from VPA group were subjected to behavioral developmental test as mentioned previously ${ }^{15}$ and were separated into ASD group $(n=10)$ and ASD + rapamycin (ASD + RAPA) group $(n=10)$. The postnatal first day was recorded as P1. The offspring were weaned on P23. Rats in ASD + RAPA group received $4 \mathrm{mg} / \mathrm{kg}$ rapamycin $(20 \mathrm{mg} / \mathrm{mL}$ in ethanol, diluted in $0.9 \% \mathrm{NaCl}$ ) with stomach perfusion every day since P24, respectively. Both normal group and ASD group were treated with the same amount of physiological saline. After 10 days, rats were killed and hippocampal tissues were prepared for further experiments. The animal study was approved by the Animal Care and Use Committee of Maternal and Children Hospital of Tangshan city.

\section{Growth and development test Weight measurement}

The weights of pups from control group and VPA group were measured on P7, P9, P14, and P21, respectively.

\section{Negative geotaxis}

Pups were placed on a head down position on a $25^{\circ}$ inclined surface, and the time for pups to complete a $180^{\circ}$ turn was recorded once on $\mathrm{P} 7, \mathrm{P} 9, \mathrm{P} 14$, and $\mathrm{P} 21$, respectively.

\section{Nociceptive perception test}

Nociceptive effects were evaluated using thermal paw withdrawal tests on $\mathrm{P} 7, \mathrm{P} 9, \mathrm{P} 14$, and $\mathrm{P} 21$, respectively. Rats were gently placed on hot plate $\left(55^{\circ} \mathrm{C} \pm 0.5^{\circ} \mathrm{C}\right)$, and the time when the rats on the plates start to lick postpedes was defined as nociceptive threshold.

\section{Social behavior}

The test was carried out under red light and unfamiliar test cage. The test cage was illuminated by a $40 \mathrm{~W}$ red light. The rats were kept individually for $3.5 \mathrm{~h}$ prior to the experiment. On the day of experiment, two rats from the same group but different litters and cages were placed into the test cage with 
$60 \times 40 \times 40 \mathrm{~cm}^{3}(1 \times \mathrm{w} \times \mathrm{h})$ for $15 \mathrm{~min}$. The frequency and duration of pairs, chasing companions, and physical contact were observed and recorded.

\section{Repetitive and exploratory activity}

A $100 \times 100 \times 40 \mathrm{~cm}^{3}(1 \times \mathrm{w} \times \mathrm{h})$ plastic container with a transparent glass cover was prepared for the test of repetitive and exploratory activity. Each rat on P35 from normal, ASD, or ASD + RAPA group was preplaced into the container for 10 min to adapt the condition, and then was moved to the central region. The total straight times and duration time of grooming was noted for $10 \mathrm{~min}$.

\section{Cognitive ability detection}

Morris water maze test was performed to evaluate the pups' learning and memory abilities according to the details. ${ }^{16}$ During the test, the lights and the placement of rats were unchanged in order to avoid interference of environmental factors.

\section{Spatial navigation}

The apparatus consisted of a black circular tank $(160 \mathrm{~cm}$ in diameter, $60 \mathrm{~cm}$ in height) filled to a depth of $40 \mathrm{~cm}$ with water, and the temperature of water was controlled at $24^{\circ} \mathrm{C} \pm 1^{\circ} \mathrm{C}$. The pool was theoretically divided into four equal quadrants. A rat was randomly placed in one of the quadrants into the pool. Then the time taken by the rat to find the platform was recorded as escape latency. If the rat failed to find the platform within $120 \mathrm{~s}$, it was manually guided to the platform and allowed to remain on it for $20 \mathrm{~s}$. Then the escape latency was recorded as $120 \mathrm{~s}$. Animals had three trials every day at an interval of $15 \mathrm{~min}$, and the average of three trials was the average escape latency.

\section{Space exploration experiment}

When the spatial navigation trials were completed, the escape platform was removed, and the rat was allowed to swim freely in the tank for $120 \mathrm{~s}$. The number of crossing platform (cross number) and the time spent in the target quadrant was calculated.

\section{Immunohistochemistry and TUNEL staining}

Hippocampal pyramidal neurons (area CA1) was isolated and fixed with $10 \%$ freshly prepared ice cold paraformaldehyde, embedded in paraffin wax, and cut into $3 \mathrm{~mm}$ thick sections. The sections were incubated with $3 \%$ hydrogen peroxide in methanol for $15 \mathrm{~min}$ in order to block the activity of endoperoxidase. After being incubated with bovine serum albumin (Sigma-Aldrich Co.) to block the nonspecific sites, the slides were incubated with primary anti-BDNF (Santa Cruz Biotechnology Inc., Santa Cruz, CA, USA; sc-546, 1:500) or anti-Bcl-2 (Santa Cruz Biotechnology Inc.; sc-783, 1:500) antibodies at room temperature. After $2 \mathrm{~h}$, the slides were washed with phosphate-buffered saline and incubated with biotinylated anti-rabbit secondary antibody (Vector Laboratories, Inc., Burlingame, CA, USA; BA-4001) for another $2 \mathrm{~h}$. The antibody binding sites were visualized through the reaction with diaminobenzidine $-\mathrm{H}_{2} \mathrm{O}_{2}$ solution. Five high-powered fields were randomly chosen by using an inverted microscope. Positive cells were stained in brown or dark nankeen. The positive rate was quantified with ImageJ software (NIH, Bethesda, MD, USA) by counting the average optical density.

TUNEL staining was performed to visualize apoptotic cells using the TUNEL Kit (Roche, Indianapolis, IN, USA) according to the manufacturer's protocols. Briefly, after being deparaffinized, the sections were incubated with proteinase $\mathrm{K}$ and permeabilized with $0.5 \%$ Triton $\mathrm{X}-100$. Then the sections were incubated with TUNEL reaction mixture for $60 \mathrm{~min}$ and were stained with DAPI. For each sample, five non-overlapping fields at $200 \times$ magnification in sections were randomly captured using a light microscope (Nikon Corporation, Tokyo, Japan). Apoptotic index $(\mathrm{AI})=$ (apoptotic cells total cells) $\times 100 \%$.

\section{Western blot}

Approximately 50-100 mg of rat hippocampal tissues were extracted and lysed with RIPA lysis buffer. The homogenate was centrifuged at $4^{\circ} \mathrm{C}$ for $10 \mathrm{~min}$ at $6,000 \mathrm{rpm}$. The supernatant was collected for western blot analysis. Protein concentration was determined by a bicinchoninic acid protein assay kit (Beyotime Biotechnology Company, Shanghai, People's Republic of China). Approximately $50 \mu \mathrm{g}$ of protein was subjected to sodium dodecyl sulfate polyacrylamide gel electrophoresis and transferred onto polyvinylidene fluoride membrane. The membranes were blocked with 5\% non-fat dry milk in Tris-buffered saline containing Tween 20 for $1 \mathrm{~h}$ at room temperature, then probed with anti-BDNF (Santa Cruz Biotechnology Inc.; sc-546, 1:500) or anti-Bcl-2 (Santa Cruz Biotechnology Inc.; sc-783, 1:500) antibodies overnight at $4^{\circ} \mathrm{C}$. After overnight incubation, the blots were incubated with horseradish peroxidase-conjugated anti-rabbit secondary antibody (Santa Cruz Biotechnology Inc.; sc-2004, 1:2,000) for $2 \mathrm{~h}$ at room temperature and subsequently developed with enhanced chemiluminescence solution according to the manufacturer's instructions. Bands were visualized using Gel imaging system (Bio-Rad, Hercules, CA, USA) and quantified using Quantity One software (Bio-Rad). The target protein band density was normalized with GAPDH (Santa Cruz Biotechnology Inc.; sc-25778, 1:250). 


\section{Statistical analysis}

All the experiments were performed at least three times using independent samples. All analytical data were expressed in mean values \pm standard deviation (SD). Statistical analysis was conducted using Statistical Package for the Social Sciences (SPSS) 17.0 software (SPSS Inc., Chicago, IL, USA). The level of significance was determined through the $P$-value. In all statistical analyses, $P<0.05$ was considered significant.

\section{Results}

VPA exposure affected the body weight, negative geotaxis, and nociceptive threshold

Various tests were performed to examine the effect of VPA on rats. First, the body weights of pups from control group and VPA group on P7, P9, P14, and P21 were measured. As shown in Table 1, compared with the control group, VPA exposure decreased the body weight of pups on P7, P9, P14, and $\mathrm{P} 21$ with significant difference $(P<0.01)$. Then oblique plate experiment was performed on $\mathrm{P} 7, \mathrm{P} 9, \mathrm{P} 14$, and $\mathrm{P} 21$ in order to detect negative geotaxis. Rats from VPA group took more time to complete a $180^{\circ}$ turn than those in control group (Table 2; $P<0.01$ ). Hot plate test was used to evaluate the nociceptive effects of pups on P7, P9, P14, and P21 (Table 3). The nociceptive threshold in VPA group was higher than that in control group with significant difference $(P<0.01)$. These results showed that VPA exposure to prenatal rats affected the body weight, negative geotaxis, and nociceptive threshold of the pups, suggesting VPA successfully induced the ASD model in the experiment.

\section{Rapamycin adjusted the social behavior and repetitive and exploratory activity of VPA-induced ASD rats}

To determine the effect of rapamycin on ASD, rapamycin was given to pups of VPA exposure group with stomach perfusion, and the social behavior was observed (Table 4). Compared with normal group, ASD group rats showed a decrease in frequency and duration of social behavior and the straight

Table I Weight measurement of rats in control group and ASD group $(\bar{x} \pm s d, n=8, g)$

\begin{tabular}{lllll}
\hline Groups & P7 & P9 & PI4 & P2 I \\
\hline Control & $14.18 \pm 1.59$ & $16.43 \pm 1.29$ & $25.32 \pm 2.76$ & $37.39 \pm 3.42$ \\
VPA & $9.87 \pm 1.12^{* *}$ & $10.93 \pm 1.10 * *$ & $16.13 \pm 1.62 * *$ & $30.54 \pm 3.05 * *$ \\
\hline
\end{tabular}

Note: Compared with control group, $* * P<0.01$.

Abbreviations: VPA, valproic acid; ASD, autism spectrum disorder; sd, standard deviation.
Table 2 Oblique plate tests of control and ASD groups $(\bar{x} \pm s d$, $\mathrm{n}=8, \mathrm{~s}$ )

\begin{tabular}{lllll}
\hline Groups & P7 & P9 & PI4 & P2 I \\
\hline Control & $154.20 \pm 10.62$ & $87.89 \pm 6.42$ & $42.15 \pm 4.31$ & $27.39 \pm 3.42$ \\
VPA & $228.43 \pm 5.37 * *$ & $136.43 \pm 6.80 * *$ & $81.18 \pm 5.69 * *$ & $66.13 \pm 4.80 * *$ \\
\hline
\end{tabular}

Note: Compared with control group, ${ }^{* * P}<0.01$.

Abbreviations: VPA, valproic acid; ASD, autism spectrum disorder; sd, standard deviation.

times, but an increase in grooming frequency $(P<0.01)$. ASD + RAPA group increased the frequency and duration of social behavior and the straight frequency, but decreased the grooming duration compared with ASD group $(P<0.01)$. However, the frequency and duration of social behavior, the straight and grooming frequency of ASD + RAPA group had no significant difference with the normal group $(P>0.05)$. These data revealed that rapamycin obviously alleviated social problems and enhanced the exploration ability of VPA-induced ASD.

\section{Rapamycin treatment protected against memory deficit induced by VPA exposure}

In order to examine the effect of rapamycin on learning and memory abilities of rats, Morris water maze test was performed (Table 5). Compared with normal group, the average escape latency of ASD group was extended, and the number of crossing platform (cross number) was significantly decreased $(P<0.01)$. However, the average escape latency of ASD + RAPA group was obviously shortened compared with ASD group $(P<0.01)$ but had no difference with normal group $(P>0.05)$. The number of crossing platform in ASD + RAPA group were increased compared with ASD group $(P<0.01)$, but had no difference with normal group $(P>0.05)$. These results showed that rapamycin mitigated memory deficit of VPA exposureinduced ASD rats.

\section{Rapamycin suppressed hippocampal neuron apoptosis in VPA-induced} ASD rats

In order to detect the influence of rapamycin on hippocampal neuron, the hippocampal tissues were isolated and TUNEL assay was performed (Figure 1A). Little hippocampal neurons of normal group and RAPA group was apoptotic.

Table 3 Hot plate tests in different groups $(\bar{x} \pm s d, n=8, s)$

\begin{tabular}{lllll}
\hline Groups & P7 & P9 & PI4 & P2I \\
\hline Control & $42.94 \pm 3.86$ & $33.94 \pm 2.86$ & $13.15 \pm 2.79$ & $8.28 \pm 1.06$ \\
VPA & $69.29 \pm 4.6 I^{* *}$ & $63.13 \pm 5.4 I^{* *}$ & $28.92 \pm 2.48 * *$ & $14.97 \pm 1.09 * *$ \\
\hline
\end{tabular}

Note: Compared with control group, $* * p<0.01$.

Abbreviations: VPA, valproic acid; sd, standard deviation. 
Table 4 Social behaviors in different groups $(\bar{x} \pm s d, n=8)$

\begin{tabular}{|c|c|c|c|c|}
\hline \multirow[t]{2}{*}{ Groups } & \multicolumn{2}{|c|}{ Social behaviors } & \multicolumn{2}{|c|}{ Repetitive and exploratory activity } \\
\hline & Frequency & Duration (s) & Straight times & Grooming (s) \\
\hline Normal & $35.64 \pm 3.28$ & $63.44 \pm 1.79$ & $26.99 \pm 2.70$ & $97.42 \pm 9.80$ \\
\hline ASD & $18.55 \pm 1.86^{* *}$ & $34.89 \pm 3.90 * *$ & $7.58 \pm 2.90 * *$ & $236.38 \pm 24.57^{* *}$ \\
\hline ASD + RAPA & $32.83 \pm 3.04^{\# \#}$ & $60.13 \pm 6.07^{\# \#}$ & $24.49 \pm 2.50^{\#}$ & $105.43 \pm 10.14^{\# \#}$ \\
\hline
\end{tabular}

Notes: ASD + RAPA, offspring from ASD rats were injected with rapamycin. Compared with normal group, ${ }^{* * P}<0.0 \mathrm{I}$; compared with $A S D$ group, ${ }^{\# P}<0.01$.

Abbreviations: ASD, autism spectrum disorder; RAPA, rapamycin; sd, standard deviation.

The AI of ASD group was significantly increased than that of normal group $(P<0.01)$. However, AI in ASD + RAPA group was lower than that in ASD group and was similar to that in normal group (Figure 1B). These results showed that ASD was related to hippocampal neuron apoptosis, and rapamycin inhibited hippocampal neuron apoptosis in VPAinduced ASD rats.

\section{Rapamycin treatment released the expression of $\mathrm{Bcl}-2$ and BDNF in VPA- induced $A S D$ rats}

Bcl-2 and BDNF were closely associated with apoptosis. The expression of Bcl-2 and BDNF were examined to investigate the mechanism of rapamycin suppressing hippocampal neurons apoptosis. Figure $2 \mathrm{~A}$ and $\mathrm{B}$ shows that the signals of Bcl-2 and BDNF were observed in hippocampus of the rats in normal or RAPA group. In ASD group, the signals were significantly weaker than those in normal group $(P<0.01)$. The positive rates of $\mathrm{Bcl}-2$ and BDNF in ASD + RAPA group were improved than those in ASD group. The positive rates of $\mathrm{Bcl}-2$ and $\mathrm{BDNF}$ in different groups were quantified by ImageJ software (Table 6). In addition, the similar results were found in western blot. Figure $3 \mathrm{~A}$ and $\mathrm{B}$ shows that the protein levels of Bcl-2 and BDNF were suppressed in ASD group, but the levels showed no significant difference between ASD + RAPA group and normal group $(P>0.05)$. These data indicated that rapamycin improved the expression of $\mathrm{Bcl}-2$ and BDNF to suppress hippocampus apoptosis induced by VPA exposure.

Table 5 Morris water maze tests of different groups $(\bar{x} \pm s d, n=8)$

\begin{tabular}{lll}
\hline Groups & Average escape latency (s) & Cross number \\
\hline Normal & $12.48 \pm 1.20$ & $2.39 \pm 2.33$ \\
ASD & $33.29 \pm 3.32^{* *}$ & $0.86 \pm 0.06^{* *}$ \\
ASD + RAPA & $13.07 \pm 1.38^{\#}$ & $2.27 \pm 0.02^{\#}$ \\
\hline
\end{tabular}

Notes: ASD + RAPA, offspring from ASD rats were injected with rapamycin. Compared with normal group, ${ }^{* * P}<0.01$; compared with ASD group, ${ }^{\# P} P<0.01$. Abbreviations: ASD, autism spectrum disorder; RAPA, rapamycin; sd, standard deviation.

\section{Discussion}

\section{Rapamycin treatment mitigated social behavior abnormality and improved the learning and memory in VPA-induced ASD rats}

Prenatal exposure to VPA is closely associated with high incidence of ASD; hence, VPA is widely utilized to induce ASD animal models. ${ }^{17}$ In this context, VPA was injected in female rats on E12.5 in order to construct a reliable rat model for ASD according to the reference. ${ }^{18}$ Social behavior is a primary deficit in ASD. According to various behavior tests, such as weight measurement, negative geotaxis, and nociceptive perception test, we found that prenatal VPA exposure successfully induced ASD-like behavior defect. This experiment provided new evidence for the practicability of prenatal VPA administration inducing ASD. The success of ASD modeling supplied the reliable experimental animal model for subsequent research about ASD.

TSC-mediated inhibition of mTOR signal is required not only for development but also for proper function of the adult brain. The disinhibited mTOR contributes to ASD-related phenotypes. ${ }^{19}$ Rapamycin is an Food and Drug Administration-approved immunosuppressant drug that inhibits activity of the mTOR protein kinase in the rodent brain following peripheral administration. ${ }^{20}$ The knockdown of TSC1/2 activated mTOR and generated ASD, ${ }^{7}$ and the supplement of rapamycin mitigated ASD-like behavior and synaptic pruning deficits. ${ }^{21}$ Based on these previous research studies, this study aimed to investigate the effect of rapamycin on the social behavior and the impairment of hippocampus in ASD models.

ASD is characterized by deficits in social interaction, language and communication impairments, and repetitive behaviors. In the study of social behavior test, compared with ASD group, rapamycin treatment increased the frequency and duration of social behavior and the straight frequency, but decreased the grooming duration, suggesting that rapamycin improved the interest of ASD rats in new things and reduced 


\section{A}

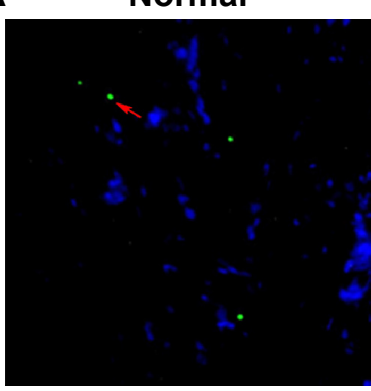

ASD

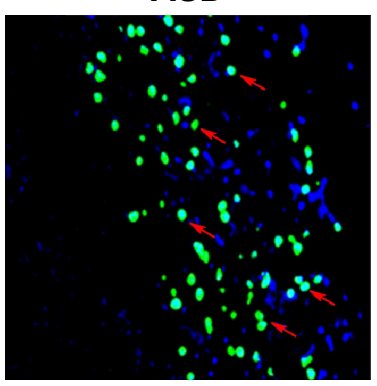

RAPA

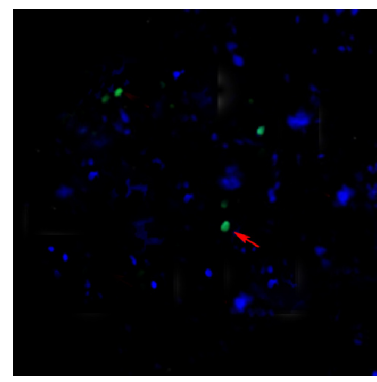

ASD + RAPA

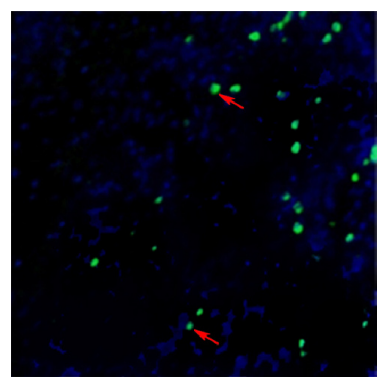

B

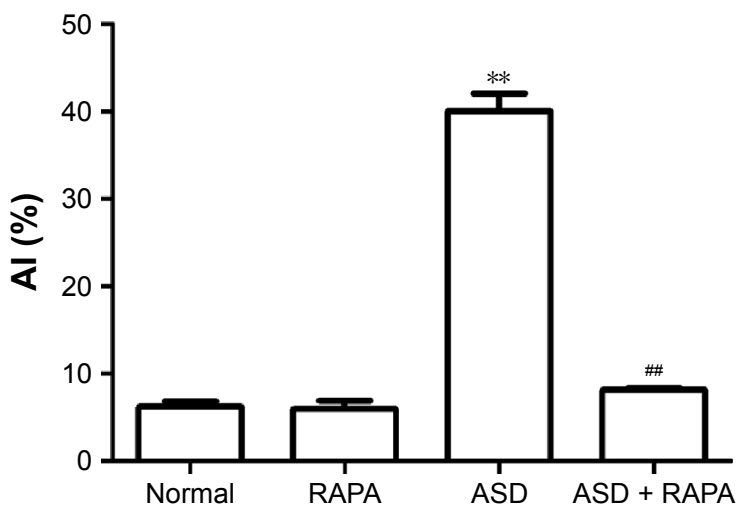

Figure I Rapamycin repressed the hippocampus apoptosis induced by VPA.

Notes: (A) Hippocampus apoptosis detected by TUNEL assay. Hippocampal tissues were isolated from different groups and analyzed by TUNEL Kit. Apoptotic cells were stained in green (indicated by red arrows). The cells were captured under 200× magnification. (B) Apoptotic index of hippocampal CAI region in different groups. For each sample, five non-overlapping fields at 200x magnification in sections were randomly captured using a light microscope. RAPA group, the offspring of control rats were administered with rapamycin; ASD + RAPA group, the offspring of VPA rats were injected with rapamycin. Apoptotic index $(A \mathrm{I})=($ apoptotic cells/total cells $) \times 100 \%$.

Compared with normal group, ${ }^{* * P}<0.01$; compared with ASD group, ${ }^{\# P}<0.01$.

Abbreviations: VPA, valproic acid; RAPA, rapamycin; ASD, autism spectrum disorder.

A

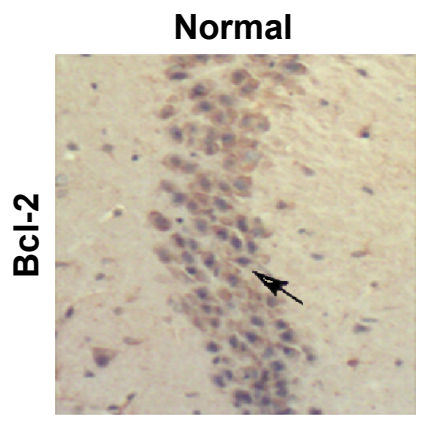

B

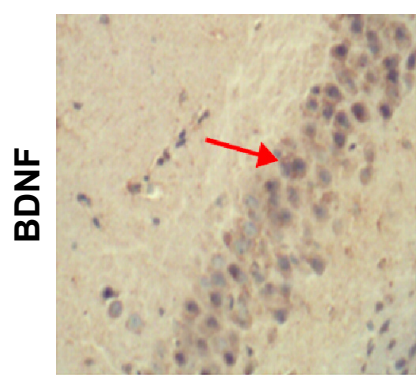

RAPA
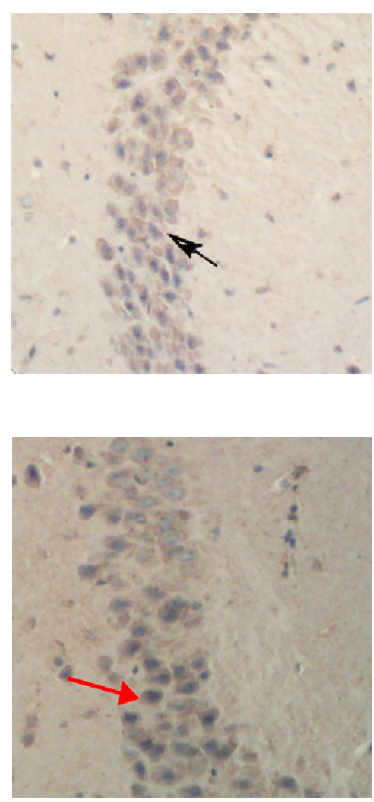

ASD
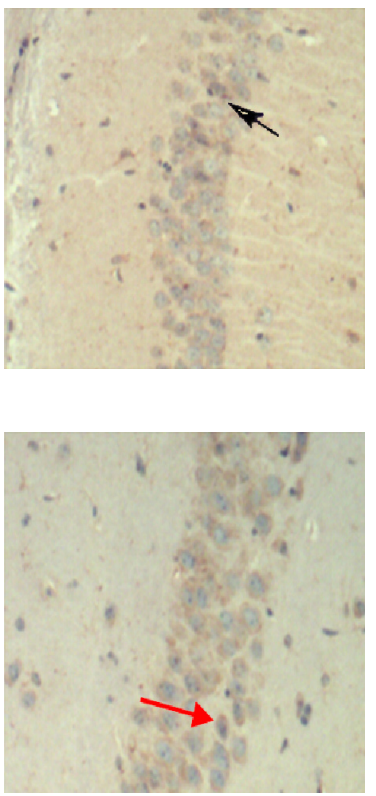

ASD + RAPA
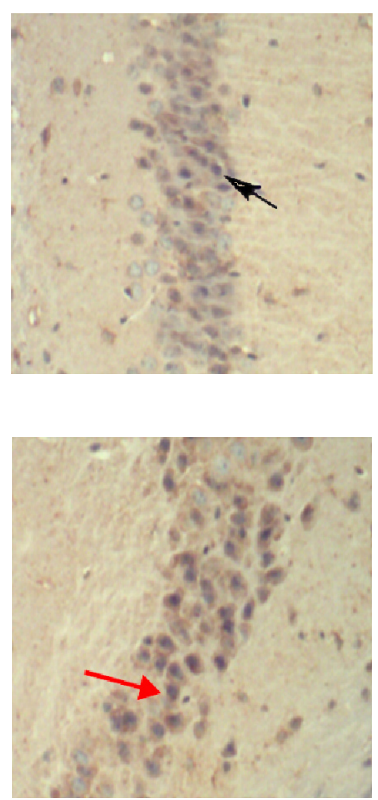

Figure 2 The expression of $\mathrm{Bcl}-2$ and BDNF was examined by immunohistochemistry.

Notes: Signals of Bcl-2 (A) and BDNF (B) in hippocampus from different groups were analyzed by immunohistochemistry. Five high-powered fields were randomly chosen for analysis under 200× magnification using an inverted microscope. The positive cells were stained in brown or dark nankeen (marked with arrows). RAPA group, the offspring of control rats were administered with rapamycin; ASD + RAPA group, the offspring of VPA rats were injected with rapamycin. The positive rate was quantified with ImageJ software by counting the average optical density.

Abbreviations: Bcl-2, B-cell lymphoma 2; BDNF, brain-derived neurotrophic factor; VPA, valproic acid; RAPA, rapamycin; ASD, autism spectrum disorder. 
Table 6 Signals of $\mathrm{Bcl}-2$ and BDNF in different groups $(\bar{x} \pm s d$, $\mathrm{n}=8, \%)$

\begin{tabular}{lll}
\hline Groups & Bcl-2 & BDNF \\
\hline Normal & $1.77 \pm 0.20$ & $1.41 \pm 0.12$ \\
RAPA & $1.65 \pm 0.45$ & $1.59+0.24$ \\
ASD & $0.85 \pm 0.10^{* *}$ & $0.59 \pm 0.08^{* *}$ \\
ASD + RAPA & $1.68 \pm 0.24^{\# \#}$ & $1.38 \pm 0.2 \mathrm{I}^{\#}$ \\
\hline
\end{tabular}

Notes: RAPA group, rats from control group were treated with rapamycin. ASD + RAPA, rats from VPA group were treated with rapamycin. Compared with normal group, ${ }^{* *} P<0.01$; compared with ASD group, ${ }^{\#} P<0.01$.

Abbreviations: Bcl-2, B-cell lymphoma 2; BDNF, brain-derived neurotrophic factor; VPA, valproic acid; RAPA, rapamycin; ASD, autism spectrum disorder; sd, standard deviation.

the repetition of stereotyped behaviors. Ozonoff et al reported that ASD patients have defects in spatial memory ability. ${ }^{22}$ In the Morris water maze experiments, compared with ASD group, rapamycin supplement reduced the average escape latency and increased the frequency of crossing platform, revealing that rapamycin improved the learning and memory ability. Taken together, rapamycin mitigated the social
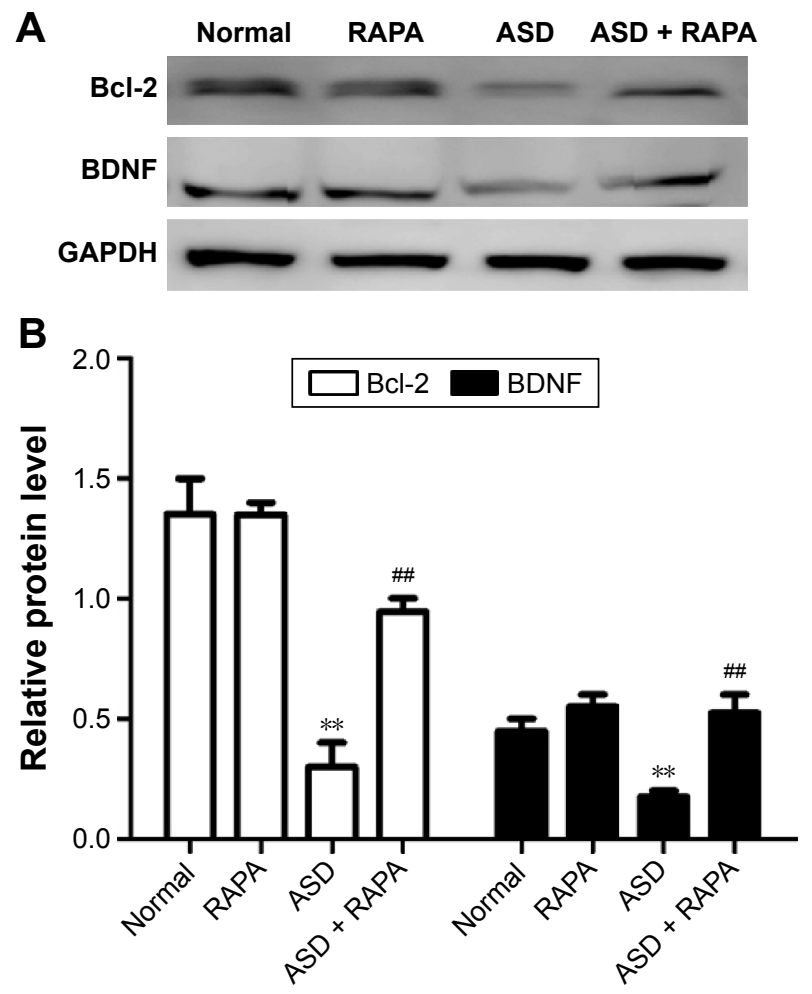

Figure 3 The protein levels of $\mathrm{Bcl}-2$ and BDNF from different groups were detected by western blot.

Notes: (A) Rapamycin released the levels of $\mathrm{Bcl}-2$ and BDNF suppressed by VPA exposure. Proteins of hippocampus were extracted for western blot. (B) Bands were quantified by using Quantity One software. RAPA group, the offspring of control rats were administered with rapamycin; ASD + RAPA group, the offspring of VPA rats were injected with rapamycin. Compared with normal group, $* * P<0.01$; compared with ASD group, $\#<<0.01$.

Abbreviations: Bcl-2, B-cell lymphoma 2; BDNF, brain-derived neurotrophic factor; VPA, valproic acid; RAPA, rapamycin; ASD, autism spectrum disorder. behavior abnormality and improved the learning and memory of VPA-induced ASD rats.

\section{Rapamycin increased the expression of $\mathrm{Bcl}-2$ and BDNF to suppress the hippocampus apoptosis in VPA-induced model}

Hippocampus is a brain region crucial for memory formation and sensitive to external damage. The central cognitive processing function is critically dependent on the hippocampus. ASD is postulated to be the developmental syndrome of hippocampal neuron dysfunction. ${ }^{23}$ Some reports showed that the impairment in memory and learning was related to hippocampal apoptosis. ${ }^{24}$ This study found that the hippocampal AI in ASD group was higher than that in normal group, which was in agreement with the previous study; ${ }^{24}$ however, rapamycin repressed the AI. These data provided new evidence that the incidence of ASD was associated with hippocampal neuron apoptosis.

Cell apoptosis is regulated by apoptosis-related proteins. Anti-apoptotic protein Bcl-2 is involved in apoptosis pathway by heterodimerization with Bax. ${ }^{25}$ Some evidence shows that the expression of Bcl-2 was suppressed in VPA-induced ASD rats. ${ }^{26}$ BDNF plays an important role in survival and differentiation of nervous system, and BDNF is involved in the pathogenesis of a wide range of psychiatric disorders. ${ }^{27}$ The levels of BDNF and Bcl-2 were decreased in ASD patients. ${ }^{28,29}$ In VPA-induced ASD rats, the expressions of BDNF, AKT, and Bcl-2 were suppressed. ${ }^{24}$ The present study found that the expression of Bcl-2 and BDNF in ASD group was lower than that in normal group, which was similar to previous reports. In early life epilepsy and ASD patients, rapamycin treatment decreased the expression of $\mathrm{p}-4 \mathrm{E}-\mathrm{BP} 1$ and activated BDNF and mitigated old life epilepsy and autistic-like behavior. ${ }^{30}$ In our subject, the protein levels of Bcl-2 and BDNF were released in ASD + RAPA group than those in ASD group. Taken together, rapamycin upregulated the expression of Bcl-2 and BDNF to promote the survival of hippocampus and to improve the learning and memory ability in ASD.

\section{Conclusion}

In summary, VPA-induced ASD rats were successfully modeled according to various behavior tests. Rapamycin supplement resulted in the increase in the protein levels of Bcl-2 and BDNF and repressed the hippocampal apoptosis, which in turn improved the social behavior and learning 
and memory ability. This study revealed the significant pathogenesis of ASD and provided new insight of rapamycin on improving ASD social behavior. This investigation may contribute to the development of new therapeutic strategies.

\section{Disclosure}

The authors report no conflicts of interest in this work.

\section{References}

1. Spitzer RL, Williams JB. Diagnostic and Statistical Manual of Mental Disorders. Arlington County: American Psychiatric Association; 1980 [Citeseer].

2. Newschaffer CJ, Croen LA, Daniels J, et al. The epidemiology of autism spectrum disorders. Annu Rev Public Health. 2007;28:235-258.

3. Kazdoba TM, Leach PT, Yang M, et al. Translational Mouse Models of Autism: Advancing Toward Pharmacological Therapeutics; 2016; $28: 1-52$.

4. Nie D, Sahin M. A genetic model to dissect the role of Tsc-mTORC1 in neuronal cultures. Methods Mol Biol. 2012;821:393-405.

5. LaSarge CL, Danzer SC. Mechanisms regulating neuronal excitability and seizure development following mTOR pathway hyperactivation. Front Mol Neurosci. 2014;7:18.

6. Chong ZZ, Shang YC, Wang S, Maiese K. A critical kinase cascade in neurological disorders: PI3K, Akt and mTOR. Future Neurol. 2012;7(6):733-748.

7. Tsai PT, Hull C, Chu Y, et al. Autistic-like behaviour and cerebellar dysfunction in Purkinje cell Tsc1 mutant mice. Nature. 2012;488(7413): 647-651.

8. Kim D-H, Sarbassov DD, Ali SM, et al. mTOR interacts with raptor to form a nutrient-sensitive complex that signals to the cell growth machinery. Cell. 2002;110(2):163-175.

9. Fletcher L, Evans TM, Watts LT, Jimenez DF, Digicaylioglu M. Rapamycin treatment improves neuron viability in an in vitro model of stroke. PLoS One. 2013;8(7):e68281.

10. Liu K, Shi N, Sun Y, Zhang T, Sun X. Therapeutic effects of rapamycin on MPTP-induced Parkinsonism in mice. Neurochem Res. 2013;38(1):201-207.

11. Siman R, Cocca R, Dong Y. The mTOR inhibitor rapamycin mitigates perforant pathway neurodegeneration and synapse loss in a mouse model of early-stage alzheimer-type tauopathy. PLoS One. 2015;10(11):e0142340.

12. Burket JA, Benson AD, Tang AH, Deutsch SI. Rapamycin improves sociability in the BTBR T+ Itpr3 tf/J mouse model of autism spectrum disorders. Brain Res Bull. 2014;100:70-75.

13. Boucher J, Mayes A, Bigham S. Memory in autistic spectrum disorder. Psychol Bull. 2012;138(3):458.

14. Schneider T, Przewłocki R. Behavioral alterations in rats prenatally exposed to valproic acid: animal model of autism. Neuropsychopharmacology. 2005;30(1):80-89.
15. Omer VES, Ali S, Holson R, et al. Behavioral and neurochemical effects of prenatal methylenedioxymethamphetamine (MDMA) exposure in rats. Neurotoxicol Teratol. 1991;13(1):13-20.

16. Wang Q-Q, Zhu L-J, Wang X-H, et al. Chronic trigeminal nerve stimulation protects against seizures, cognitive impairments, hippocampal apoptosis, and inflammatory responses in epileptic rats. J Mol Neurosci. 2016;59(1):78-89.

17. Castro K, Baronio D, Perry IS, Riesgo RdS, Gottfried C. The effect of ketogenic diet in an animal model of autism induced by prenatal exposure to valproic acid. Nutr Neurosci. 2016 Epub Feb 9.

18. Silvestrin RB, Bambini-Junior V, Galland F, et al. Animal model of autism induced by prenatal exposure to valproate: altered glutamate metabolism in the hippocampus. Brain Res. 2013;1495:52-60.

19. Ehninger D, Silva AJ. Rapamycin for treating tuberous sclerosis and autism spectrum disorders. Trends Mol Med. 2011;17(2):78-87.

20. Meikle L, Pollizzi K, Egnor A, et al. Response of a neuronal model of tuberous sclerosis to mammalian target of rapamycin (mTOR) inhibitors: effects on mTORC1 and Akt signaling lead to improved survival and function. $J$ Neurosci. 2008;28(21):5422-5432.

21. Tang G, Gudsnuk K, Kuo S-H, et al. Loss of mTOR-dependent macroautophagy causes autistic-like synaptic pruning deficits. Neuron. 2014;83(5):1131-1143.

22. Ozonoff S, Young GS, Landa RJ, et al. Diagnostic stability in young children at risk for autism spectrum disorder: a baby siblings research consortium study. J Child Psychol Psychiatry. 2015;56(9):988-998.

23. Qin L, Dai X, Yin Y. Valproic acid exposure sequentially activates Wnt and mTOR pathways in rats. Mol Cell Neurosci. 2016;75:27-35.

24. Gao J, Wang X, Sun H, et al. Neuroprotective effects of docosahexaenoic acid on hippocampal cell death and learning and memory impairments in a valproic acid-induced rat autism model. Int $J$ Dev Neurosci. 2016;49:67-78.

25. Borkan SC. The role of BCL-2 family members in acute kidney injury. Semin Nephrol. 2016;36(3):237-250.

26. Kim J-E, Shin M-S, Seo T-B, et al. Treadmill exercise ameliorates motor disturbance through inhibition of apoptosis in the cerebellum of valproic acid-induced autistic rat pups. Mol Med Rep. 2013;8(2):327-334.

27. Zheleznyakova GY, Cao H, Schiöth HB. BDNF DNA methylation changes as a biomarker of psychiatric disorders: literature review and open access database analysis. Behav Brain Funct. 2016;12(1):1.

28. Halepoto DM, Bashir S, Zeina R, Al-Ayadhi LY. Correlation between hedgehog $(\mathrm{Hh})$ protein family and brain-derived neurotrophic factor (BDNF) in autism spectrum disorder (ASD). J Col Phys Surg. 2015; 25(12):882-885.

29. Ghanizadeh A. Malondialdehyde, Bcl-2, superoxide dismutase and glutathione peroxidase may mediate the association of sonic hedgehog protein and oxidative stress in autism. Neurochem Res. 2012; 37(4):899-901.

30. Talos DM, Sun H, Zhou X, et al. The interaction between early life epilepsy and autistic-like behavioral consequences: a role for the mammalian target of rapamycin (mTOR) pathway. PLoS One. 2012; 7(5):e35885.
Neuropsychiatric Disease and Treatment

\section{Publish your work in this journal}

Neuropsychiatric Disease and Treatment is an international, peerreviewed journal of clinical therapeutics and pharmacology focusing on concise rapid reporting of clinical or pre-clinical studies on a range of neuropsychiatric and neurological disorders. This journal is indexed on PubMed Central, the 'PsycINFO' database and CAS,

\section{Dovepress}

and is the official journal of The International Neuropsychiatric Association (INA). The manuscript management system is completely online and includes a very quick and fair peer-review system, which is all easy to use. Visit http://www.dovepress.com/testimonials.php to read real quotes from published authors. 